\title{
Techniques for Extracting Region of Interest in Breast Cancer
}

\author{
Veena M, Rashmi A R
}

\begin{abstract}
The main aim of the project is to develop an automatic system which detects the stages and Region of Interest (ROI) in Breast images. Breast cancer in females is one of the main causes for deaths among women. Finding at early stage of breast cancer which helps in the treatment and recovery rates. Discovering region of interest for breast in the breast images is a demanding problem. Retrieving of breast tumors region and the pectoral muscle is a key step in the process of CAD.
\end{abstract}

Keywords: Breast Cancer, Region of Interest (ROI), DBT images, Segmentation.

\section{INTRODUCTION}

The breast cancer is the serious issue of that affects women. It is one of the major causes of death in women. Early detection of breast cancer plays an essential role in successful treatment. Digital Breast Tomosynthesis (DBT) is a 3D mammography which eliminates the overlapping tissues problem. It over comes the limitations of the conventional mammogram where it takes the breast images by rotating the tube over the breast to get multiple $\mathrm{x}$-ray picture which detect the small tumor that hidden in a conventional mammogram. It uses a series of 2D images to build a 3Dimages of the breast. The advantages of DBT are it reduces the rate of false positive readings and used for women with dense breast.First we take the DBT images and that image is done by preprocessing. It involves several steps like image enhancement, filtering. This image is converted into gray scale image. After segmentation we obtain the ROI of the tumor in breast. In this work firstly, we separate RGB color channels, and each channel is sliced with multiple segments based on threshold. Secondly, each segment is analyzed for breast region and background region using Morphological technique to find region of interest. It includes 5 stages in Breast Cancer: stage 0(zero): there is no cancer in the breast. Stage 1: tumor is $1 \mathrm{~mm}$ to $20 \mathrm{~mm}$. Stage 2: the tumor is $>20 \mathrm{~mm}$. Stage 3 : the tumor is $<50 \mathrm{~mm}$. Stage 4 : the tumor is $>50 \mathrm{~mm}$ and spreads all over the breast.

\section{LITERATURE SURVEY}

According to paper [1] CANCER is detected using BEMD [Bi-dimensional Empirical Mode Decomposition] method. This is based on two main phases: ROI and region segmentation. In this first we are taking the image as input and then the input image is preprocessed by removing noisy element and then image is enhanced. After this image segmentation is done by using BEMD technique. BEMD plays an important role in this technique and also it improves the quality of CAD.

According to paper [2] Breast cancer is one of the most common rates in women and cause of death in many countries. In this they are used three methods: Segmentation-they have taken mammographic images as input. This image is split into red, green and blue channel. Then the each channel is sliced into five layers by giving threshold value. Intersection- The segmented layer of each layer is merged to single layer using intersection method using formula. Region of Interest-After segmentation and intersection the ROI is found by using Quad tree.

According to paper [3] The digital image processing technique is used to recognize the tumor in mammography image. The image is divided into blocks and segmentation. This is done by performing color quantization technique. After applying this technique we get the ROI. After finding ROI feature extraction is done by taking gray level co-occurrence matrix. When ROI is found the next step is to find the stages of breast cancer.

According to paper [4] Breast cancer is one of the most usual cancers in many women's throughout the world. In this MRI image we can find breast tissues like skin, glandular tissue, chest wall and fatty tissue. One of the most common is removing these tissues using tumor free model. Ultra-wide band (UWB) microwave imaging is one of the best methods for breast cancer detection.

According to paper [5] Ultra sound image is preprocessed to remove the noisy elements and the image is enhanced. After this image segmentation is done by using Hybrid ISRAD Filtering. In this we have done with two steps: Threshold and Black Hat Operation. In Black Hat Operation is used to extract detail and small elements in the image. After this segmentation ROI is found.

According to paper [6] The Mammograms image is preprocessed to remove noisy element. After Preprocessing the image segmentation is done by Artifact Suppression and background separation. In this method they have given a fixed threshold value. The next segmentation method is pectoral muscle removal. This is done by using seeded region growing method. After this segmentation ROI is found. It has been survey that the different modalities used for detecting breast cancer have some disadvantages with this context we have proposing a new modality DBT for detecting a breast cancer for a women with the dense breast.

Revised Manuscript Received on December 10, 2019.

* Correspondence Author

Veena M, Assistant Professor, Dept of CSE, PES College of Engineering Mandya. Email: mveenakemps@gmail.com

Rashmi A R, Assistant Professor, Dept of CSE, PES College of Engineering Mandya. 


\section{RESULTS AND DISCUSSIONS}

\section{Image Preprocessing}

In first step of preprocessing we take the input DBT images, and remove the noisy element, resize the image. This step is done by using with related commands.

The commands used for preprocessing are

Table 1: Pre-processing Functions

\begin{tabular}{|l|l|}
\hline imread & Input image \\
\hline imcrop & Crop image \\
\hline imresize & Resize image \\
\hline imrotate & Rotate image \\
\hline imtool & To study image pixel \\
\hline rgb2gray & Converting image to gray scale \\
\hline imadjust & Image enhancement \\
\hline
\end{tabular}

\section{Image segmentation}

After preprocessing the image we do segmentation using OTSU method. To remove backgrounds we use Morphological function. After removing the background in the image we find the tumor and ROI is applied to that tumor. Comparing with other methods OTUS's is quite good for our project. OTSU algorithm is an easy form which split the pixel into 2 category, foreground and background. To get output image with the same size compare to the input image to use morphological operation.

\section{Feature selection}

In feature extraction we use GLCM (gray level co-occurrence matrix). This algorithm consists of several parameters like area, shape, texture and density features.

To get exact result in biomedical image it is required that biomedical image must be good quality. Hence we are using GLCM algorithm. There are some GLCM features with the formula they are listed below:

Table 2: Feature Extraction Formulae

\begin{tabular}{|c|c|c|}
\hline SI.No & GLCM Feature & Formula \\
\hline 1. & Contrast & $\sum_{i, j=0}^{N-1} P_{i, j}(i-j)^{2}$ \\
\hline 2. & Correlation & $\sum_{i, j=0}^{N-1} P_{i, j}\left[\frac{\left(i-\mu_{i}\right)\left(j-\mu_{j}\right)}{\sqrt{\left(\sigma_{i}^{2}\right)\left(\sigma_{j}^{2}\right)}}\right]$ \\
\hline 3. & Dissimilarity & $\sum_{i, j=0}^{N-1} P_{i, j}|i-j|$ \\
\hline 4. & Energy & $\sum_{i, j=0}^{N-1} P_{i, j}^{2}$ \\
\hline 5. & Entropy & $\sum_{i, j=0}^{N-1} P_{i, j}\left(-\ln P_{i, j}\right)$ \\
\hline 6. & Homogeneity & $\sum_{i, j=0}^{N-1} \frac{P_{i, j}}{1+(i-j)^{2}}$ \\
\hline 7. & Mean & $\mu_{i}=\sum_{i, j=0}^{N-1} i\left(P_{i, j}\right), \mu_{j}=\sum_{i, j=0}^{N-1} j\left(P_{i, j}\right)$ \\
\hline 8. & Variance & $\sigma_{i}^{2}=\sum_{i, j=0}^{N-1} P_{i, j}\left(i-\mu_{i}\right)^{2}, \sigma_{j}^{2}=\sum_{i, j=0}^{N-1} P_{i, j}\left(j-\mu_{j}\right)^{2}$ \\
\hline 9. & Standard Deviation & $\sigma_{i}=\sqrt{\sigma_{i}^{2}}, \quad \sigma_{j}=\sqrt{\sigma_{i}^{2}}$ \\
\hline
\end{tabular}

4. classification
The designed algorithms make use of ensemble of classifier. After finding the ROI, the stages of the cancer is detected. The tumor size is calculated in terms of centimeters (cm). The stages of the tumor are given below:

T0 ( $\mathrm{T}$ zero): no cancer in the breast.

T1: $20 \mathrm{~mm}$ or smaller in size.

T2: $20 \mathrm{~mm}-50 \mathrm{~mm}$.

T3: $>50 \mathrm{~mm}$.

T4: It can be any size. In this stage the tumor spreads all over the breast.

When compare to other classifier Multi SVM place an important role in our project. Instead of using two or more different classifier we use a single multi SVM classifier, which plays an important role to find the stages in breast cancer.

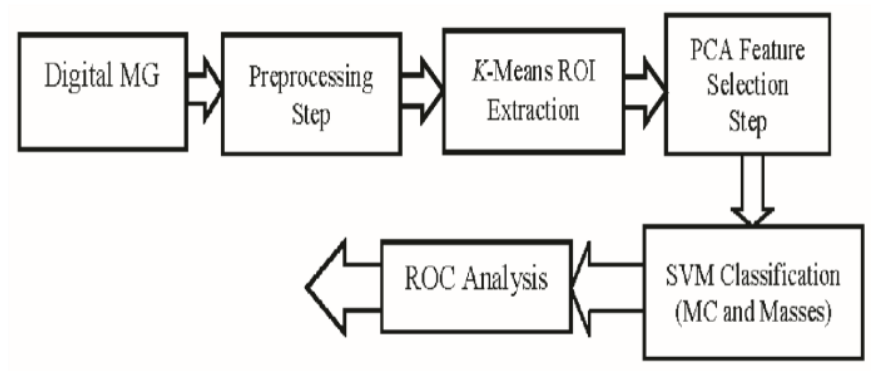

Figure1: BLOCK DIAGRAM

\section{CONCLUSION}

As per literature survey, compared to the other modalities DBT works well especially for the women with dense breasts. DBT images useful in diagnostic and therapeutic applications. It enables the doctor to locate accurately where the tumor is present in the breast.

\section{REFERENCES}

1. Early Breast Cancer Detection By Using Image Processing. Author: Kamini T Waghulade and Prof. Ajit Kumar Yadav. 26th June, 2016[PAPER ID: ICITER-D184]

2. Novel approach to locate region of interest in mammograms for Breast cancer. Author : B V Divyashree, Amarnath R, Naveen M, G Hemanth Kumar.IJISAE,2018 PUBLISHED:29/09/2018

3. A Novel Approach for Detection of Breast Cancer at an early stage using Digital Image processing techniques, Author : Sangeetha R, Dr. Srikanta Murthy K. (ICISC-2017) 2017IEEE.

4. Early Breast Cancer Detection via UWB Based on MRI-derived Model. Author: Xia Xiao, Qinwei Li, Liang Wang. IEEE.

5. A Novel Algorithm for Locating Region Of Interest in Breast Ultra Sound Images. Author: Stafford Michahial and Bindu A Thomas. 2017ICEECCOT.

6. Automated Breast Profile segmentation for ROI detection using digital mammograms. Author: Jawad Nagi, Sameem Adbul Kareem, Farrukh Nagi, Syed Khaleel Ahmed. (IECBES 2010)

7. Subash Chandra Bose, K. Shankar Kumar, M.,Karnan. "Detection of Microcalcification in Mammograms using Soft Computing Techniques," European Journal of Scientific, vol. 86, no. 1, pp.103-122, 2012

8. KAMINI T WAGHULADE, AJIT KUMAR YADAV, "Early Breast Cancer Detection By Using Image Processing" IJIERT-ICITER-16,ISSN:2394-3696 Pg no 147-151 .

9. Jawad Nagi, Sameem Abdul Kareem, Farrukh Nagi, Syed Khaleel Ahmed, "Automated Breast Profile Segmentation for ROI Detection Using Digital Mammograms" IECBES 2010, Pg no 87-92

10. C. DeSantis, K. Beaver, G. J. Ma, L Bryan, \& A. Jemal, "Breast cancer statistics, 2013," A Cancer Journal for Clinicians, vol.64, no.1, pp.52-62, 2014 
11. S. C. Bose, K. Shankar Kumar, M. Karnan, "Detection of Microcalcification in Mammograms using Soft Computing Techniques," European Journal of Scientific, vol. 86, no. 1, pp.103-122. 2012.

12. Subash Chandra Bose, K. Shankar Kumar, M.,Karnan. "Detection of Microcalcification in Mammograms using Soft Computing Techniques," European Journal of Scientific, vol. 86, no. 1, pp.103-122, 2012.

13. G. Boccignone, A. Chianese, A. Picariello, "Computer aided detection of micro-calcifications in digital mammograms," Comput. Biol Med, vol.30, no.5, pp.267-286, 2000.

14. R. Nakayama, Y. Uchiyama, K. Yamamoto, "Computer-aided diagnosis scheme using a filter bank for detection of micro-calcifications clusters in mammograms. IEEE Trans Biomed Eng., vol.53, no.2, pp.273-283, 2006.

15. A. Papadopoulos A, Fotiadis DI, Likas A: An automatic microcalcifications detection system based on a hybrid neural network classifier. ArtifIntellMed, vol.25, no.2, pp.149-167, 2002.

16. L. Li, W. Qian, L.P. Clarke, R.A. Clark, J.A. Thomas, "Improving mass detection by adaptive and multiscale processing in digitized mammograms," Proc. of SPIE 3661, Medical Imaging: Image Processing, pp. 490-498. 1999.

\section{AUTHORS PROFILE}

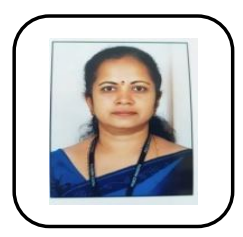

First Author: Veena M

Assistant Professor,

Department of CSE,

PES College of Engineering, Mandya. Area of interest is Image Processing.I am persuing my $\mathrm{PhD}$ in Medical image processing.

Mail id-mveenakemps@gmail.com

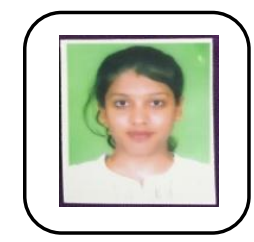

Second Author: Rashmi A R,

M.Tech, Dept of CSE.

PES College of Engineering Mandya,

Mail id- rojanandi26@gmail.com. 